\title{
Dentists' professional behavioural characteristics
}

\author{
Janine Brooks, Associate Director of Dentistry, \\ National Patient Safety Agency
}

Send your comments to the

Editor-in-Chief,

British Dental Journal

64 Wimpole Street,

London

W1G 8YS

Email bdj@bda.org
There is increasing awareness of the importance of the behavioural characteristics of clinical professionalism and it is now the turn of the dental profession to be considered in this light.

The time has come for the profession to celebrate the characteristics which combine to make a dental practitioner. We all have our own ideas about what a dentist looks like and how they behave, but that is just the point as these are our own subjective opinions and there is little or no actual evidence behind what we think or perceive. We need the evidence. Unlike medicine, where there is a substantial amount of information about behavioural characteristics, relatively little is known about the personality and attitudinal traits of dentists and how these traits impact on their performance.

\section{SUBSTANTIAL KNOWLEDGE GAP}

To fill this substantial knowledge gap we need to gather information from dentists across the UK. Once these behavioural traits are defined, then we can consider how they influence the practice of individual dentists. Dentistry owes itself the privilege of building a greater understanding of factors that affect the profession and combine to produce the dentists that currently work in the UK.

There is some curiosity about the possibilities that a study of behavioural characteristics can bring to groups such as dental schools and those involved in foundation training. For example, some dental schools already use psychometric tests as part of their selection process. We in the National Clinical Assessment Service (NCAS) work with employers/commissioners, dentists and other organisations to support dentists (and doctors and pharmacists) who are experiencing difficulties, and struggling in their day to day practice. We aim to help them back to valued, safe practice. Sometimes we undertake assessments of dentists and one component of this appraisal is a behavioural evaluation. This helps us to identify the values and beliefs that are important to the dentist and which underpin their attitudes and behaviour. This in turn helps us to understand what may be contributing to the difficulties they are experiencing, to agree an appropriate plan to address them and support the practitioner to get back on track.

\section{GREATER NUMBERS OF DENTISTS NEEDED}

Relatively few dentists undergo an NCAS assessment. However, we are keen to know if the characteristics found in the dentists we assess are common across the dental profession or if there are differences. To help us answer this question we are calling for volunteers to join a study which will map out key behavioural characteristics for dentists. In doing so, each person who takes part will learn more about themselves and that knowledge may help them in their professional lives.

NCAS has recently commissioned a pilot study of 100 volunteer dentists. This has revealed some useful information, but we need greater numbers for the results to be valid. Preliminary results show, for example, that the dentists who volunteered tended to be more positive and collaborative and more compassionate than most people. We also found that they tended to be more susceptible and reactive to stress, and that they were less optimistic and less assertive than the wider UK working population. This is not only interesting in its own right but may perhaps run quite contrary to accustomed thinking.

We are now working with Edgecumbe Consulting to extend the study. Volunteer dentists will be invited to complete two online questionnaires (the NEO PI-R and the Hogan Development Survey). These will take up to 60 minutes to complete in total and participants will receive a feedback overview of their personal profile. The individual results will, of course, be confidential and will be used only in an amalgamated and anonymised form with all personal information deleted. No individual data will be passed to NCAS.

Our hope is that this will be a really useful piece of work and that the results will benefit the profession in many ways. It will provide a better understanding of the behavioural norms of dentists and will enable NCAS and the BDA to develop their services for dentists more appropriately. We are hopeful that the results of the study will be published in the $B D J$ in due course.

The study has the support of the BDA, which is encouraging dentists to participate. More information about the study is available on the BDA website at www.bda.org where you can also contact Edgecumbe Consulting about participating.

\section{ABOUT NCAS}

NCAS provides a range of services to healthcare organisations concerned about the performance of a dentist, doctor or pharmacist. www.ncas.npsa.nhs.uk

Contact: Janine Brooks, Associate Director for Dentistry at janine.brooks@ncas.npsa.nhs.uk.

DOI: $10.1038 / s j . b d j .2010 .3$ 